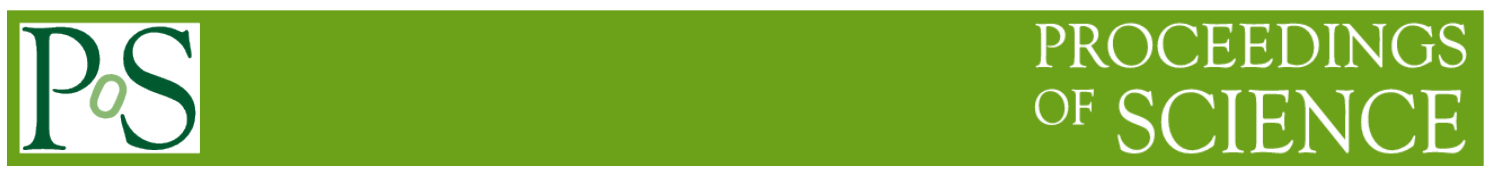

\title{
Models for cosmic ray transport in the era of AMS-02
}

\author{
Simon Kunz ${ }^{1}$ \\ Karlsruhe Institute of Technology \\ Institut für Experimentelle Kernphysik \\ Wolfgang-Gaede-Str. 1 \\ D-76131 Karlsruhe \\ E-mail: Simon.Kunz@kit.edu \\ Iris Gebauer \\ Karlsruhe Institute of Technology \\ Institut für Experimentelle Kernphysik \\ Wolfgang-Gaede-Str. 1 \\ D-76131 Karlsruhe \\ E-mail: Iris.Gebauer@kit.edu
}

\begin{abstract}
State-of-the-art models for galactic cosmic ray transport as implemented in the DRAGON or GALPROP codes describe a multitude of observations. However, recent measurements of electrons, positrons and protons by AMS-02 and PAMELA challenge our understanding of cosmic ray sources and the subsequent transport processes in the interstellar medium. Here, we use Markov chain Monte Carlo methods to investigate wide ranges of transport parameters. Solutions to the transport equation are numerically obtained by using the DRAGON code. A total amount of more than 15 million solutions was generated. The predictions are compared to measurements of cosmic ray nuclei using data from PAMELA, ACE, CREAM, ISOMAX and HEAO. More than 13,000 models were found to have a maximum deviation from the data of 1 sigma averaged over all data points. We find that even in low dimensional models no definite solution exists. Based on the models found in our analysis, we predict the flux of secondary positrons, produced in proton-gas interactions, and compare our prediction to the AMS-02 data. We determine the local flux of additional energetic positrons necessary to describe the data. We further discuss different transport scenarios, which can explain the change of slope of the proton spectrum at high rigidities, and comment on the discriminating power of future data on B/C. The method described here will be applied to the upcoming AMS-02 data in the future.
\end{abstract}

The 34th International Cosmic Ray Conference

30 July- 6 August, 2015

The Hague, The Netherlands

${ }^{1}$ Speaker 


\section{Introduction}

Galactic cosmic rays (CRs) are believed to be produced predominantly in supernova remnants (SNRs) throughout the Milky Way [1]. Upon injection into the interstellar medium (ISM) they scatter off magnetic turbulences, leading to a random walk which can be modeled by diffusion. In addition, diffusive reacceleration, convection, particle losses via escape from the galaxy and energy losses via ionisation, Coulomb losses, synchrotron radiation and Bremsstrahlung modify the initial CR spectra. These processes can be described by a diffusion equation, as implemented in the publicly available GALPROP [2] and DRAGON [3] codes. Both programs solve the diffusion equation in steady-state, using a realistic treatment of the galactic gas distribution, the magnetic field and the interstellar radiation field.

We study global transport processes by a comparison of model predictions to experimental data of hadronic observables. While energetic leptons in the range of hundreds of $\mathrm{GeV}$ quickly lose energy via synchrotron radiation and inverse Compton losses, nuclei have larger energy loss times and transport lengths of the order of kpc. Therefore, the parameters deduced from hadronic observables correspond to the averaged ones throughout the Galaxy. The considered hadronic observables include the locally measured proton and antiproton spectrum and the ratios $\overline{\mathrm{p}} / \mathrm{p}, \mathrm{B} / \mathrm{C}$ and ${ }^{10} \mathrm{Be} /{ }^{9} \mathrm{Be}$. While the proton spectrum yields information about the source spectrum and the diffusion process, the antiproton spectrum and the ratios $\mathrm{B} / \mathrm{C}$ and ${ }^{10} \mathrm{Be} /{ }^{9} \mathrm{Be}$ allow to constrain the CR interaction rate and the CR escape time.

In a preceding study the relevant ranges of transport parameters of a 16-dimensional model setup were identified by using Markov chain Monte Carlo (MCMC) techniques [5]. In total, more than 15 million sets of transport parameters have been evaluated by an efficient sampling of the parameter space. Many models were found to describe the data. In general, the data prefer models with an unbroken nuclei injection spectrum and low convection velocities. We reduced the degrees of freedom and derived a model with only six free parameters.

Here, we focus on this inferred minimal model. We show that even in such low dimensional models some of the major transport parameters cannot be pinned down due to strong correlations and wide ranges of transport parameters are compatible with the data.

In chapter 2 the examined, minimal model and its parameters are introduced. The results, based on 840,610 numerically evaluated models, are presented in chapter 3 . We discuss the role of the particular observables in terms of their constraining power and their sensitivities on the particular transport parameters. The limits of a model selection consisting of more than 13,000 data-conform models with strongly differing parameter combinations are presented and the parameter-induced uncertainty on the expected flux of secondary produced positrons is quantified (chap. 3.3). In chap. 4, we discuss two scenarios which can explain the hardening of the proton spectrum as measured by AMS-02.

\section{Transport model setup}

Our model depends on 6 free transport parameters which describe the nuclei injection spectrum, diffusion, diffusive reacceleration and the halo height, beyond which free escape of the particles is assumed. The nuclei injection spectrum is assumed to be a power law in momentum 
$\operatorname{dp}(\mathrm{q}) / \mathrm{dp} \propto \mathrm{p}^{-\alpha}$ and is assumed to be equal for all nuclei species. The applied SNR source distribution was proposed by Yuan et al. [4] and it is a good compromise to other, more extreme distributions proposed in the literature. Based on a selection of several hundred models, we assured that the choice of the SNR distribution has a negligible influence on the results.

The spatial diffusion coefficient is taken to be constant and isotropic in the Galaxy. It is parameterized by

$$
D(\rho)=D_{0} \beta^{\eta}\left(\frac{\rho}{\rho_{0}}\right)^{\delta},
$$

with $\rho=\mathrm{p} /(\mathrm{Ze})$ the rigidity of the particle of charge $\mathrm{Z}$ and momentum $\mathrm{p}$. The normalization is given by the diffusion constant $\mathrm{D}_{0}$ in units of $10^{28} \mathrm{~cm}^{2} / \mathrm{s}$. The rigidity dependence, given by $\delta$, corresponds to the level of turbulence, relevant for resonant scattering at the corresponding rigidity. The reference rigidity $\rho_{0}$ is fixed to the commonly used value $\rho_{0}=4 G V$. The parameter $\eta$ determines the low energy behaviour of the diffusion coefficient. The strength of diffusive reacceleration, corresponding to diffusion in momentum space, is determined by the Alfvén velocity $\mathrm{v}_{\alpha}$.

In addition, four parameters accounting for the solar modulation of CR particles are included. The solar modulation is modeled by the force-field approximation [6]. Since its strength is strongly model dependent, the effective modulation potentials are fitted to the corresponding data sets after the particles have been propagated. The potentials $\Phi$ are allowed to be different for data sets recorded during different epochs of the solar activity as well as for oppositely charged particles [7].

The solution to the transport equation is numerically obtained in a cylindrical model using the DRAGON code. Along the galactocentric radius the grid resolution is set to $\Delta \mathrm{r}=1 \mathrm{kpc}$ and along the perpendicular direction to $\Delta \mathrm{z}=0.2 \mathrm{kpc}$, independent of the assumed halo height $\mathrm{L}$.

Table 1 lists the transport parameters and their respective limits for the MCMC sampling. The considered observables and data sets are p, $\overline{\mathrm{p}}, \overline{\mathrm{p}} / \mathrm{p}$ (PAMELA [8],[9]), B/C (ACE[10], CREAM[11], ISOMAX[12]) and ${ }^{10} \mathrm{Be} /{ }^{9} \mathrm{Be}(\mathrm{HEAO}[13]$, ISOMAX[12]).

\begin{tabular}{|c|c|c|c|}
\hline Parameter & Unit & Lower limit & Upper limit \\
\hline $\mathrm{D}_{0}$ & $10^{28} \mathrm{~cm}^{2} / \mathrm{s}$ & $10^{-3}$ & $10^{4}$ \\
$\mathrm{~L}$ & $\mathrm{kpc}$ & 0.25 & 30.0 \\
$\delta$ & 1 & 0.0 & 1.2 \\
$\eta$ & 1 & -2.0 & 2.0 \\
$\mathrm{~V}_{\mathrm{a}}$ & $\mathrm{km} / \mathrm{s}$ & 0.0 & 70.0 \\
$\mathrm{a}$ & 1 & 0.0 & 3.0 \\
\hline$\Phi_{\mathrm{p}}$ & $\mathrm{MV}$ & 0.0 & 1000.0 \\
$\Phi_{\overline{\mathrm{p}}}$ & $\mathrm{MV}$ & 0.0 & 1000.0 \\
$\Phi_{\mathrm{B}, \mathrm{C}}$ & $\mathrm{MV}$ & 0.0 & 1000.0 \\
$\Phi_{\mathrm{Be}}$ & $\mathrm{MV}$ & 0.0 & 1000.0 \\
\hline
\end{tabular}

Table 1: The 6 transport parameters and their selected limits. The lower panel shows the 4 modulation potentials used in the force-field-approximation which are fitted for each model to the corresponding data after propagation.

A model's goodness is quantified by a $\chi^{2}$ value. Since the particular observables are sensitive to different transport parameters, each observable's $\chi^{2}$ value is normalized to the corresponding number of data points to ensure a fair, i.e. equitable, weighting. 


\section{Results}

In total, 840,610 parameter combinations were efficiently sampled in the minimal model by MCMC methods. While large step sizes were used to cover the whole parameter space to be analyzed, small step sizes were additionally used to focus on interesting regions. The predictions of wide and continuously connected ranges of transport parameters are found to be in agreement with the data. The degenerated solutions can consequently not be distinguished by the available data on locally measured nuclei energy spectra. In the following, we show which observables yield the strongest constraining power on the transport parameters.

\subsection{Relevant parameter ranges}

The strongest constraints for all transport parameters result from the proton spectrum and B/C . The weakest constraints come from the ${ }^{10} \mathrm{Be} /{ }^{9} \mathrm{Be}$ measuements which suffer from large uncertainties by which ${ }^{10} \mathrm{Be} /{ }^{9}$ Be plays here a minor part.

Fig. 1 shows the obtained minimal $\bar{\chi}^{2}$ per number of degrees of freedom (Ndof), illustrated by a projection of transport parameters on binned, 2-dimensional planes. Clear borders in the planes of $\mathrm{D}_{0}-\mathrm{L}$ and $\alpha-\delta$ are visible. Regions outside the colored planes were found to be excluded. The halo height $\mathrm{L}$ deprives from a reasonable restriction upwards and only low values of $\mathrm{L}<$ $0.85 \mathrm{kpc}$ can be excluded. A monotonic relationship between $\mathrm{D}_{0}$ and $\mathrm{L}$, expressed by the Spearman's rank coefficient, was found to be 0.99 .

Even though the proton spectrum fixes the combination of $\alpha$ and $\delta$, it is not suitable for limiting the particular parameters. A lower and upper limitation of $\delta$ is forced by $\mathrm{B} / \mathrm{C}$ resulting in the combined limitation of $\alpha$ and $\delta$. Within the preferred range, we find those two parameters to be strongly and linearly anticorrelated with -0.98 .

\subsection{Model selection}

In order to quantify the allowed parameter ranges, a selection on the 840,610 sampled models is applied. Among the best fit model, we find another 13,400 models, characterized by yielding a maximal average deviation of $1 \sigma$ to each data set, i.e. on average each data point of observable $j$ is matched within the experimental uncertainty:

$$
\chi_{\mathrm{j}}^{2} / \mathrm{N}_{\mathrm{j}}^{\mathrm{d}} \leqslant 1 \text {. }
$$

Fig. 2 shows the energy spectra and ratios of the best fit model and each model selected according to equation (2). The parameter limits of these models and the values of the best fit model are listed in table 2. Also listed are the parameter limits derived from a subset of 10,050 models including constraints from the secondary-to-primary ratio $\mathrm{SubFe} / \mathrm{Fe}$ with $\mathrm{SubFe}=$ $\mathrm{Sc}+\mathrm{Ti}+\mathrm{V}$. This observable constrains the parameters $\delta, \alpha$ and $\eta$.

The range of the diffusion constant could be constrained to $\left(0.58 \leq \mathrm{D}_{0} \leq 8.55\right) 10^{28} \mathrm{~cm}^{2} / \mathrm{s}$. While the best data description was obtained for a rather low halo height of $\mathrm{L}=2.15 \mathrm{kpc}$, halo heights up to the maximal tolerated value of $\mathrm{L}=30 \mathrm{kpc}$ cannot be excluded due to strong and unresolvable parameter correlations. The rigidity dependence of the diffusion coefficient, $\delta$, was found to be data-compatible in the range $0.39 \leq \delta \leq 0.71$ and in the range $0.49 \leq \delta \leq 0.68$ if constraints from SubFe/Fe are taken into account. A Kolmogorov spectrum $(\delta=1 / 3)$ is therefore disfavoured and the results point to an Iroshnikov- Kraichnan spectrum with $\delta=1 / 2$. 
Compatible Alfvén velocities were found ranging from $\mathrm{v}_{\alpha}=0 \mathrm{~km} / \mathrm{s}$ up to $20 \mathrm{~km} / \mathrm{s}$ and are upper bound by the proton spectrum and B/C. The best fit model yields $\mathrm{v}_{\alpha} \approx 10 \mathrm{~km} / \mathrm{s}$. The range of the spectral index of the nuclei injection spectrum is $2.11 \leq \alpha \leq 2.47$, but is much stronger constrained if $\mathrm{SubFe} / \mathrm{Fe}$ is taken into account, leading to $2.14 \leq \alpha \leq 2.36$. It should be noted, that this additional contraint only applies, if it is assumed that all nuclei have the same injection index as protons.

The model predictions for heavier nuclei (specifically ${ }^{3} \mathrm{He},{ }^{4} \mathrm{He},{ }^{26} \mathrm{Al} /{ }^{27} \mathrm{Al},{ }^{54} \mathrm{Fe} /{ }^{56} \mathrm{Fe}, \mathrm{O} / \mathrm{C}$ ) are in agreement with the currently available measurements. However, the latter are not precise enough to further constrain any parameter. More precise data on the nuclei spectra are expected by AMS-02 and ISS-CREAM in the near future.

\begin{tabular}{|c|c|c|c|c|c|c|}
\hline Parameter & Unit & Lower limit & Upper limit & Lower limit* & Upper limit* & Best fit \\
\hline $\mathrm{D}_{0}$ & $10^{28} \mathrm{~cm}^{2} / \mathrm{s}$ & 0.58 & 8.55 & 0.58 & 7.81 & 1.79 \\
$\mathrm{~L}$ & $\mathrm{kpc}$ & 0.85 & 29.98 & 0.85 & 29.88 & 2.15 \\
$\mathrm{D}_{0} / \mathrm{L}$ & $10^{28} \mathrm{~cm}^{2} / \mathrm{s} / \mathrm{kpc}$ & 0.21 & 1.09 & 0.22 & 1.02 & 0.83 \\
$\delta$ & 1 & 0.39 & 0.71 & 0.49 & 0.68 & 0.57 \\
$\eta$ & 1 & -1.90 & 1.21 & -1.74 & 1.07 & 0.00 \\
$\mathrm{~V}_{\alpha}$ & $\mathrm{km} / \mathrm{s}$ & 0.0 & 19.90 & 0.0 & 18.65 & 10.42 \\
$\alpha$ & 1 & 2.11 & 2.47 & 2.14 & 2.36 & 2.27 \\
\hline$\Phi_{\mathrm{p}}$ & $\mathrm{MV}$ & 437 & 927 & 453 & 832 & 639 \\
$\Phi_{\overline{\mathrm{p}}}$ & $\mathrm{MV}$ & 483 & 983 & 612 & 980 & 837 \\
$\Phi_{\mathrm{B}, \mathrm{C}}$ & $\mathrm{MV}$ & 52 & 302 & 52 & 288 & 138 \\
$\Phi_{\mathrm{Be}}$ & $\mathrm{MV}$ & 0 & 1000 & 0 & 1000 & 162 \\
\hline
\end{tabular}

Table 2: Parameter ranges of the top 13,400 models. The limits were derived by demanding an average deviation to each data set of $\leq 1 \sigma$. Also shown are the limits of a subset of 10,050 models* including additional constraints from $\mathrm{SubFe} / \mathrm{Fe}$.

\subsection{Predictions for positrons}

The 13,400 selected models were used to predict the expected positron flux from nuclei interactions and to determine the discrepancy to recent measurements of the positron flux by AMS-02 [14]. For the calculation of the secondary positron production by interactions of nuclei with the ISM, most up-to-date cross section models on p-p, p-He, He-p and He-He as derived by Kamae et al. in 2006 [16] are used. For each selected model, which correctly predicts the CR interaction rate and escape time, the expected positron flux was computed. Fig. 3 shows the uncertainty from transport parameters, mainly due to $\mathrm{D}_{0}$ and $\mathrm{L}$, as red band. A comparison between the model prediction and the data shows a significant discrepancy. In order to describe the data, an energetic positron contribution which dominates the flux of secondary positrons, as incoporated in our model, starting from energies of about $10 \mathrm{GeV}$ is required. This contribution can be calculated by a substraction of the model prediction from the data. This additional contribution is shown as the green band. The model and data uncertainties are transferred to the green band and lines, respectively. From about $15 \mathrm{GeV}$ on, the signal contribution dominates over the background expectation.

\section{The hardening of the proton spectrum}

Recently, the AMS-02 collaboration confirmed the hardening of the proton spectrum at rigidities of a few hundred GV [15] as previously measured by other space and balloon-borne 
experiments. We discuss two scenarios which explain this observation: Scenario A assumes a non-standard particle injection spectrum with a significant and abrupt decrease of the spectral index from 2.37 to 2.25 at $\rho=314 \mathrm{GV}$. Scenario B assumes an unbroken injection spectrum at high rigidities, as expected from First-order fermi acceleration in the sources, but implies different levels of magentic turbulence in the interstellar space, which give rise to a change in the rigidity dependence of the diffusion coefficient. We find that $\delta_{1}=0.49$ and $\delta_{2}=0.36$ below and above a rigidity of $\rho=330 \mathrm{GV}$ can describe the data. Fig. 4 shows the model predictions for the proton spectrum and B/C. Both scenarios describe the proton data, while slightly different predictions for $\mathrm{B} / \mathrm{C}$ are obtained. At a rigidity of $1 \mathrm{TV}$ the predictions for $\mathrm{B} / \mathrm{C}$ differ by around $13 \%$. Consequently, discriminating these scenarios by $\mathrm{B} / \mathrm{C}$ requires precise measuements at the TV scale with uncertainties comparable to the predicted discrepancy.

\section{Conclusion}

Drawing robust implications from transport models requires a precise knowledge of the underlying transport processes. Based on a less constrained preliminary study with 15 million evaluated models, we efficiently sampled 840,610 parameter combinations of a minimal model. Strong parameter correlations between major parameters have been found and quantified. A total amount of more than 13,000 different parameter combinations were sampled, all of which are able to describe the pre-AMS-02 measurements of $\mathrm{p}, \overline{\mathrm{p}}, \overline{\mathrm{p}} / \mathrm{p}, \mathrm{B} / \mathrm{C}$ and ${ }^{10} \mathrm{Be} /{ }^{9} \mathrm{Be}$.

The derived, robust limits on the transport parameters are much wider than those usually assumed, for two reasons: a) the parameter ranges considered in this study are wider than those usually assumed, specifically large halo heights of up to $30 \mathrm{kpc}$ are considered and b) the limited statistics of previous studies lead to a less thorough exploration of parameters space. In particular we find the preferred rigidity dependence of the diffusion coefficient $\delta$ to lie between 0.39 and 0.71 with a best fit value at 0.57 , which disfavors the commonly adopted Kolmogorov spectrum. The method presented here will be applied to the AMS-02 data in the future. While upcoming AMS-02 data on the spectral shape of B/C will allow to further constrain the rigidity dependence of the diffusion coefficient and thus the particle injection spectrum, the degeneracy between the diffusion constant and the halo height will not be resolved by these data.

We further discussed two explanations for the hardening of the proton spectrum at high rigidities: A change of slope due to source properties as well as a propagation induced explanation by the rigidity dependence of the diffusion coefficient. To discriminate these two explanations a B/C measurement with errors below $\sim 13 \%$ at TV rigidities would be required.
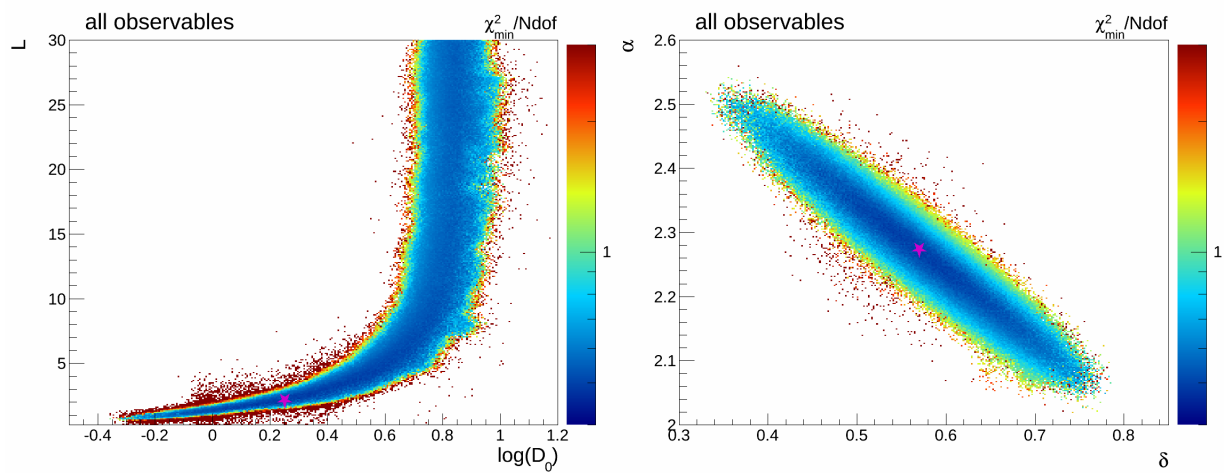

Figure 1: Minimal $\chi^{2} /$ Ndof values projected on 2-dimensional parameter planes of $D_{0}-L$ and $\delta$ - $\alpha$. Wide favoured ranges with data-conform and comparable $\chi^{2} /$ Ndof values are visible. Overlayed are the parameter values of the best-fit model marked as star. 

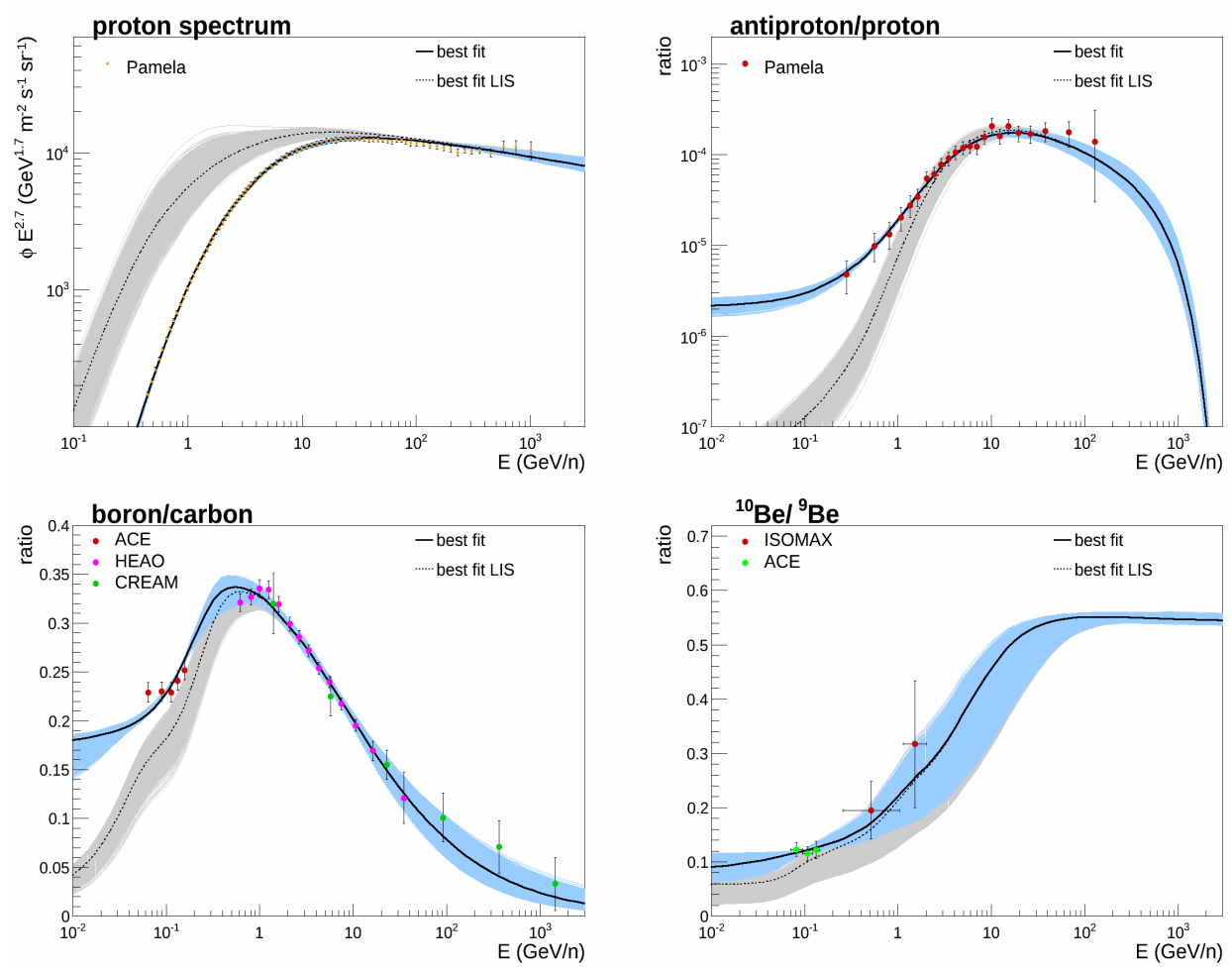

Figure 2: The energy spectra of the considered observables. Shown are the best fit model (solid black line) and a blue band corresponding the uncertainty from the transport parameters allowing for parameter correlations. The gray lines correspond to the local interstellar spectra (LIS), i.e. the spectra without correction for solar modulation induced by the solar wind.
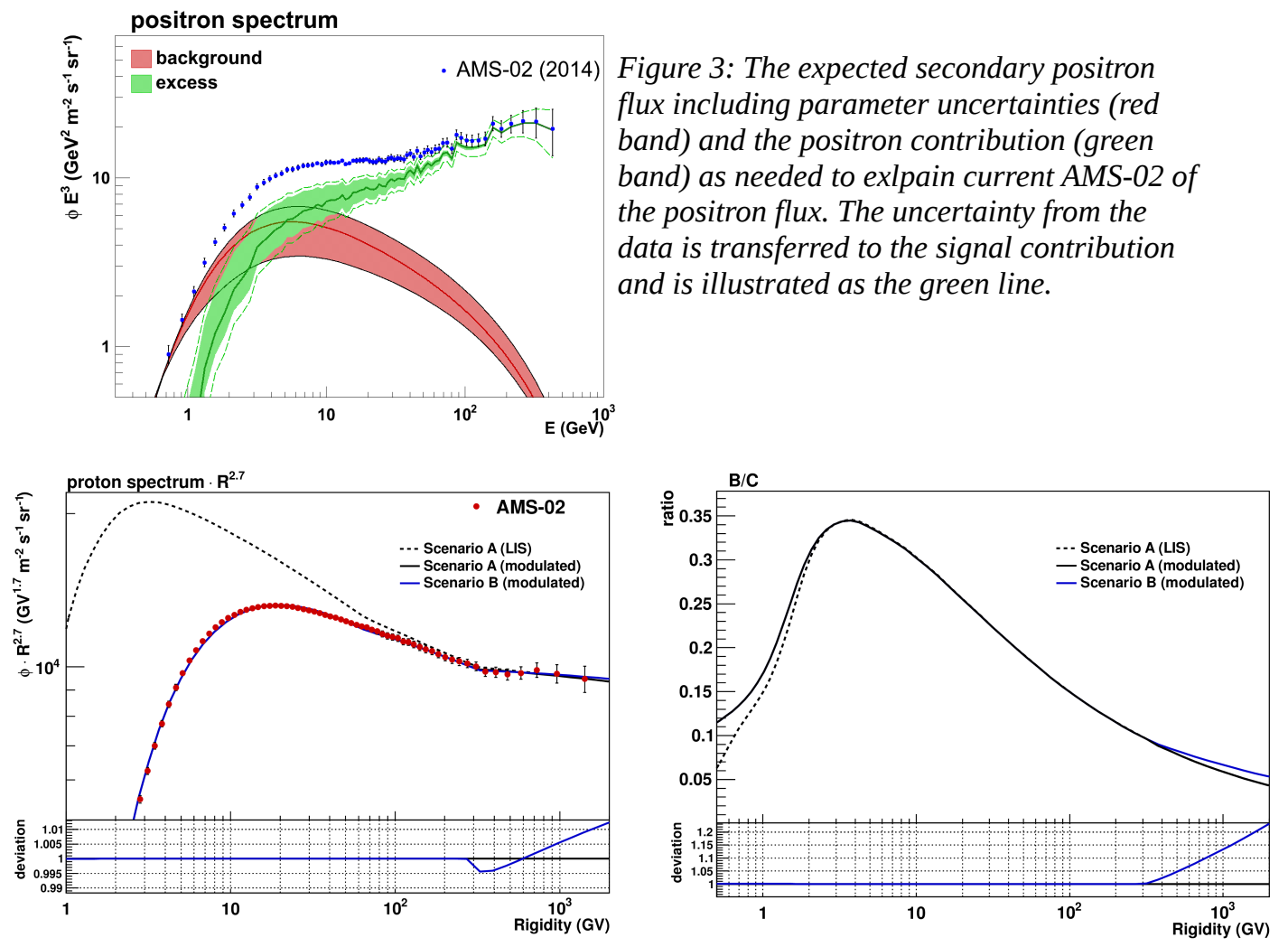

Figure 4: Model predicitions of scenarios $A$ and $B$ for the proton spectrum (left) and B/C (right). The lower panels show the deviation between the model predictions as ratios of A/A (black line) and B/A (blue line). 


\section{References}

[1] Fermi-LAT Collaboration, "Detection of the Characteristic Pion-Decay Signature in Supernova Remnants”, Science 339 (2013) 807, arXiv:1302.3307.

[2] I. Moskalenko, Modelling cosmic ray propagation and associated interstellar emissions, 39th COSPAR Scientific Assembly, 2012

[3] C. Evoli, D. Gaggero, D. Grasso et al., "Cosmic ray nuclei, antiprotons and gamma rays in the galaxy: a new diffusion model”, Journal of Cosmology and Astroparticle Physics 10, October 2008, arXiv:0807.4730.

[4] Q. Yuan, S. Liu, and X.-J. Bi, "Unified model for the gamma-ray emission of supernova remnants”, Astrophys.J. 761, 2012. arXiv:1203.0085

[5] S. Kunz, Constraints on transport models for galactic cosmic rays and their implications for the anomalous positron abundance, PhD thesis, Karlsruhe Institute of Technology, 2014. http://ekpinvenio.physik.uni-karlsruhe.de/record/48605

[6] L. J. Gleeson and W. I. Axford, “Solar Modulation of Galactic Cosmic Rays”, Astrophysical Journal 154, December 1968)

[7] H. Gast and S. Schael, “Charge-dependent solar modulation in light of the recent PAMELA data”, Proceeding to the 31st ICRC , 2009.

[8] PAMELA Collaboration, "PAMELA Measurements of Cosmic-ray Proton and Helium Spectra”, Science 332 (2011) 69-72, arXiv:1103.4055.

[9] PAMELA Collaboration, "PAMELA results on the cosmic-ray antiproton flux from $60 \mathrm{MeV}$ to 180 GeV in kinetic energy”, Phys.Rev.Lett. 105 (2010) 121101, arXiv:1007.0821.

[10] Davis et al., “AIP Conf Proc. 528, 421”, (2000).

[11] H. Ahn, P. Allison, M. Bagliesi et al., "Measurements of cosmic-ray secondary nuclei at high energies with the first flight of the CREAM balloon-borne experiment”, Astropart.Phys. 30 (2008) 133-141, arXiv:0808.1718.

[12] Hams et al., “Astrophys.J. 611, 892”, (2004).

[13] Yanasak et al., “Astrophys.J. 563, 768”, (2001).

[14] AMS Collaboration, "Electron and Positron Fluxes in Primary Cosmic Rays Measured with the Alpha Magnetic Spectrometer on the International Space Station”, Phys. Rev. Lett. 113 (Sep, 2014) 121102.

[15] AMS Collaboration, "Precision Measurement of the Proton Flux in Primary Cosmic Rays from Rigidity 1 GV to 1.8 TV with the Alpha Magnetic Spectrometer on the International Space Station”, Physical Review Letters PRL 114, 171103 (2015)

[16] T. Kamae, N. Karlsson, T. Mizuno et al., "Parameterization of Gamma, e+/- and Neutrino Spectra Produced by p-p Interaction in Astronomical Environment”, Astrophys.J. 647 (2006) 692-708, arXiv:astro-ph/0605581.

[17] bwGRiD, Member of the German D-Grid initiative, funded by the Ministry of Education and Research and the Ministry for Science, Research and Arts Baden-Württemberg.

Acknowledgment: We gratefully thank the bwGRiD project [17] for the computational resources. 